\title{
Postfaktische Zeiten
}

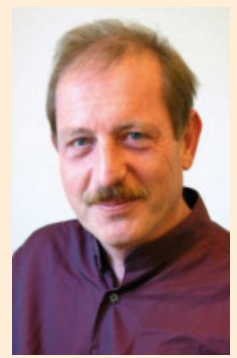

Liebe Kolleginnen und Kollegen,

die Deutsche Gesellschaft für Maritime Medizin hat einen Workshop veranstaltet, der sich mit den naheliegenden Fragen des gegenwärtigen Standes und der absehbaren Entwicklungen im maritimen Umfeld befasst hat. Das Prinzip eines Zukunftsworkshops ist nun nichts Seefahrtspezifisches und kann sinngemäß auf alle Fachgesellschaften übertragen werden.

Erstaunlich war das Ergebnis, weil eine Feststellung alles überragend war, die nichts mit zukünftigen Entwicklungen oder gar der Seefahrt im Besonderen zu tun hatte: Es fehlen faktische Informationen, an denen man längerfristig robuste Handlungsfelder ausrichten könnte. Dabei mangelt es nicht an emsiger Datenerfassung durch Forscher in der Beantwortung einer Studienfragestellung, durch Unternehmen im Rahmen ihrer Betriebsverantwortung und durch Behörden in ihrem Zuständigkeitsbereich - die Fakten sind durchaus vorhanden, irgendwo weltweit verstreut aber eben nicht zugänglich an einem Ort und damit nicht ohne Weiteres nutzbar. Die globalisierte, fragmentierte und regulationsarme Seefahrt ist hier sicherlich ein schwieriges Pflaster aber durchaus prototypisch.

Google ist, was Fakten angeht, eine Illusion, denn es liefert Fundstellen hinter denen sich tausendfach Meinungen, Manipulation, Werbung und nebenbei auch Fakten verbergen. Es ist allerdings äußerst mühsam und zeitaufwendig, Letztere aus der Informationsmasse herauszudestillieren, wenn es sich nicht um so triviale Dinge wie die Öffnungszeiten eines Restaurants handelt.

Wissenschaftliche Datenbanken liefern demgegenüber sicherlich Informationen in faktisch hoher Qualität, aber eben nur für die Dinge der Welt, die vom wissenschaftlichen Suchscheinwerfer erfasst wurden. Sie zielen aber nicht unbedingt darauf ab, Informationen für Außenstehende als alltagstaugliche rationale Entscheidungsgrundlage leichter nutzbar zu machen.

Vor diesem Hintergrund ist verständlich, dass zunehmend Teile der Bevölkerung in der Flut der Informationen die Fakten nicht mehr finden und ihnen, wenn es um die Meinungsbildung und das Entscheiden geht, der Bauch näher ist als der Kopf. Hier könnte man jetzt den Begriff „Informationsmanagement“ in den Ring werfen, doch Wikipedia sagt: „Informationsmanagement wird in der Fachliteratur unterschiedlich definiert. Das dynamische Umfeld der informationstechnischen Entwicklung und die verschiedenen wissenschaftlichen Disziplinen (insbesondere die Wirtschaftsinformatik), die sich mit Informationsmanagement (genauer: Informations- und Kommunikationsmanagement) beschäftigen, sind der Grund dafür.“

Die mögliche Lösung hat also selbst ein Problem.

Angesichts der zu beobachtenden Entwicklung sind gerade wissenschaftliche Fachgesellschaften geeignet, die Verbreitung von Fakten in zielgruppenorientierter Form zu fördern, die Informationsflut zu kanalisieren, Informationen zugänglich zu machen und alltagstaugliche Entscheidungshilfen zu geben. Hier signalisiert der Zeitgeist augenscheinlich einen steigenden Bedarf. Puzzlesteine scheint es genug zu geben, es fehlen derzeit wohl die Tische, sie sinnvoll zusammenzusetzen. Aber gerade hier bietet sich für die Arbeit der Gesellschaften neben den klassischen Veranstaltungsvarianten als Publikationsorgan, natürlich naheliegenderweise die FTR an, wenn es um den Tisch geht. 